\section{Avaliação do Programa Saúde da Família nos municípios do Estado de Santa Catarina, Brasil}

\author{
Assessment of the Family Health Program in \\ municipalities in Santa Catarina State, Brazil
}

Flávia Henrique 1

Maria Cristina Marino Calvo 1

\footnotetext{
I Programa de PósGraduação em Saúde Pública, Universidade Federal de Santa Catarina, Florianópolis, Brasil.

Correspondência M. C. M. Calvo Programa de Pós-Graduação em Saúde Pública, Universidade Federal de Santa Catarina. Campus Universitário $s / n$ Florianópolis, SC 88040-970, Brasil. mcmcalvo@ccs.ufsc.br
}

\begin{abstract}
This evaluative study aimed to classify the municipalities (counties) in the State of Santa Catarina, Brazil, as to implementation of the Family Health Program, using three categories (satisfactory, intermediate, and unsatisfactory) and two different periods (2001 e 2004). The indicators were coverage, evidence of change in the healthcare model, and impact. According to the results, coverage increased significantly from 2001 to $2004(p<0.0001)$. Evidence of change in the healthcare model and impact did not change significantly. In 2001, 48\% of the municipalities showed an unsatisfactory situation, as compared to $45 \%$ with intermediate and $7 \%$ satisfactory performance, while in 2004 the figures were 29\%, 58\%, and 13\%, respectively. Improvement in classification from 2001 to 2004 was statistically significant $(p=0.0061)$. Analysis of the data as a whole showed that changes in the final classification resulted basically from the coverage indicator, demonstrating effective expansion in access. However, these indicators did not show significant changes in the healthcare model during the period analyzed.
\end{abstract}

Family Health Program; Health Evaluation; Health Services Coverage

\section{Introdução}

A análise atenta da história e do desenvolvimento do setor saúde demonstra que não é recente a crise do modelo de atenção centrado na cura das doenças, super especializado e hospitalocêntrico, mas as propostas oriundas dessas críticas, especialmente as voltadas para o desenvolvimento do primeiro nível de atenção ou da atenção primária, têm seu surgimento atrelado às crises do sistema capitalista e aos elevados gastos sociais em saúde 1,2

Um dos pilares na construção da proposta de atenção primária em saúde foi a Conferência Internacional sobre Cuidados Primários em Saúde, em Alma Ata, no ano de 1978. Elevou-se a atenção primária em saúde como a estratégia para se alcançar a meta de Saúde para Todos no Ano 2000, definida pela assembléia mundial da Organização Mundial da Saúde (OMS), em 1977, assumindo uma proposta de extensão da cobertura dos serviços básicos de saúde com base em sistemas simplificados de assistência à saúde. A Declaração de Alma Ata, de 1978, codificou a "santidade" da atenção primária à saúde como um princípio para todos os sistemas de saúde do mundo 3 .

A proposta da atenção primária em saúde foi desenvolvida com base em diferentes concepções. Testa ${ }^{4}$ questiona sua forma de implementação, sobretudo para países subdesenvolvidos, em função da existência de serviços de saúde 
diferentes para grupos sociais também diferenciados, de forma que tal serviço não é voltado para o atendimento de toda a população; e pela contenção de custos que a atenção primária representa, sendo apresentada como alternativa à diminuição dos gastos em saúde.

Como forma de reorganizar a atenção primária no Brasil, o Ministério da Saúde lançou, em 1994, o Programa Saúde da Família (PSF). Foi em um contexto de ajuste fiscal e reformas setoriais na saúde que o programa ganhou ampla aceitação nacional, com o objetivo de superar os limites do modelo vigente, particularmente na esfera municipal 5,6.

O primeiro documento a esclarecer a forma de atuação da nova proposta foi editado pelo Ministério da Saúde em 1994, e apresentava o PSF como instrumento de reorganização da atenção à saúde respeitando os princípios do Sistema Único de Saúde (SUS), porém atendendo prioritariamente aos grupos mais vulneráveis da comunidade 5 . No entanto, em documento de 1997, o Ministério da Saúde propõe uma nova interpretação para o "saúde da família", compreendido desta vez como estratégia de atenção e não mais programa, e com capacidade de modificar o modelo assistencial vigente. Suas diretrizes operacionais indicam o caráter substitutivo do PSF, entendendo-o não como uma atividade assistencial paralela à rede implantada, mas como a porta de entrada do sistema. Neste manual estão detalhados os papéis dos membros da equipe, bem como orientações sobre a seleção, treinamento, supervisão e avaliação das equipes ? .

Nos documentos mais atuais, como Avaliação da Implementação do Programa Saúde da Família em Dez Grandes Centros Urbanos ${ }^{8}$, Avaliação Normativa do Programa Saúde da Família no Brasil 9, entre outros, percebe-se a preocupação do Ministério da Saúde com a avaliação da atenção básica no Brasil, inclusive pela criação da Coordenação de Acompanhamento e Avaliação da Atenção Básica. A principal atribuição desta coordenação é a definição de critérios, mecanismos e fluxos para avaliação da atenção básica.

Tanto quanto o Ministério da Saúde, diversos pesquisadores têm se ocupado em analisar o PSF. Os estudos de alguns desses autores são apresentados a seguir e é possível observar a evolução das análises com o desenvolvimento da proposta ao longo dos anos.

Misoczky 10 declarou, em 1994, que a proposta significava um retrocesso para o SUS, visto que, pela complexidade e perfil epidemiológico da população, seriam necessários serviços de saúde mais bem estruturados para o atendimento qualificado. Em 1994, Aguiar 11 afirma que os limites e possibilidades do PSF estariam ligadas à sua inserção nos serviços locais de saúde, podendo servir tanto como forma de simplificação da assistência quanto como motivação para mudança do modelo médico-assistencial. Para esse autor, tanto quanto para Conill 12, existiam contradições na implantação e execução do programa, com imensas variações dependentes da política municipal em que está inserido e também de sua própria concepção no nível ministerial, que vem sofrendo transformações ao longo dos anos.

Para Viana \& Dal Poz 6, em análise de 1998, o PSF era implementado preferencialmente em áreas de população carente, rural e com menor poder aquisitivo, o que na prática reflete uma política de focalização da atenção. Tal prática, associada a uma política de universalização da atenção, confirmaria a hipótese de que o PSF apresentava caráter complementar e não substitutivo.

Já em 2002, Mendes 13 coloca que o PSF, apesar de ter sofrido influências importantes do modelo inglês, canadense e cubano, é autóctone, uma vez que surge com base na experiência realizada no Ceará, tendo superado, a partir do documento de 1997, pelo menos no discurso oficial, a visão de atenção primária à saúde como um programa de atenção primária seletiva. Entretanto, Ronzani \& Stralen 14 avaliam que não há uma atuação uniforme das equipes do PSF, de forma que, mesmo com um discurso travestido de novo, o modelo de atenção individual curativa continua se perpetuando de forma hegemônica.

Dentre os estudos mais recentes sobre o PSF, destacam-se os estudos de linha de base, apoiados pela Coordenação de Acompanhamento e Avaliação do Departamento de Atenção Básica do Ministério da Saúde, e financiados pelo Banco Mundial.

Em São Paulo, Viana et al. 15 consideraram o PSF um modelo capaz de atender às populações mais vulneráveis nas regiões metropolitanas, onde outros modelos de atenção básica possuem baixa eficácia e efetividade. Complementarmente, Ibañez et al. 16 consideraram que o PSF teve melhor desempenho nas dimensões vínculo, elenco de serviços, orientação comunitária e enfoque familiar, embora possua, nas dimensões acessibilidade e porta de entrada, desempenho semelhante a outros modelos. Ainda, Elias et al. 17 relatam que, em todos os extratos sociais de usuários pesquisados, o PSF obteve melhor resultado que o modelo tradicional de unidade básica de saúde.

No Nordeste e Sul do Brasil, Fachini et al. 18 coordenaram estudo de comparação do PSF com o modelo tradicional de atenção básica, observando que o desempenho e a oferta de ações e serviços de saúde foram mais adequados no 
PSF. Contudo, verificaram que a efetivação da atenção básica de saúde é muito problemática, apresentando dificuldades no monitoramento e avaliação das ações, com supervisão apenas informativa, sobrecarga de trabalho, burocratização dos serviços e precariedade e improvisação nas estruturas das unidades locais.

Em outro estudo, Viana \& Dal Poz 6 apresentam três modelos de implantação do PSF no Brasil: modelo regional, modelo singular e modelo incipiente. O primeiro e o segundo modelos, de formas distintas, apresentam avanços no sentido da estruturação da atenção básica, enquanto o último modelo apresenta pouco desenvolvimento, demonstrando a necessidade de uma gerência do programa. Em relação aos principais problemas enfrentados, enfatizam a falta de participação de outras entidades federadas como estados e o governo federal, carência de recursos humanos e a falta de integração do PSF com outros programas municipais.

Em Santa Catarina, a implantação do PSF iniciou-se em 1994 e, desde então, convênios entre o Ministério da Saúde e os municípios foram paulatinamente assinados, ampliando o número de municípios e de equipes que participam do programa. Todavia, dados do programa surgem a partir do parecer no. 1886/GM, de 18 de dezembro de 1997, que vincula os recursos financeiros à alimentação do Sistema de Informação da Atenção Básica (SIAB) 19,20. Esse sistema congrega dados do Programa Agentes Comunitários (PACS) e do PSF e foi desenvolvido pelo Departamento de Informática do SUS (DATASUS), em conjunto com a Coordenação de Saúde Comunitária, com o intuito de acompanhar e avaliar o PSF 21,22.

Em 2005, o PSF em Santa Catarina possuía 1.053 equipes atuantes e atendia a uma população estimada em 3,3 milhões de pessoas, com uma cobertura de $59 \%$ da população catarinense 20. Apesar de vários estudos abordarem o PSF em diferentes aspectos, o estado carece de um estudo avaliativo que possibilite compreender como tem evoluído a implantação do programa.

Assim, com dados fornecidos pelo SIAB, Sistema de Informações Hospitalares (SIH) e DATASUS, este estudo desenvolveu uma proposta de avaliação da implantação do PSF entre os municípios catarinenses, analisando indicadores representativos de cobertura, indícios de mudança do modelo assistencial e impacto em dois períodos: os anos de 2001 e de 2004.

\section{Metodologia}

O método adotado foi desenvolvido valendo-se das características básicas da abordagem siste- matizada por Donabedian 23 para avaliação dos serviços de saúde. A tríade estrutura-processoresultados sistematizada pelo autor foi simplificada para indicadores representativos de cobertura potencial, indício de mudança no modelo assistencial e impacto.

Propõe-se uma pesquisa avaliativa, com dados secundários, obtidos por intermédio do SIAB e SIH, dos municípios que forneceram dados aos sistemas em 2001 e 2004.

Contandriopoulos et al. 24 sugerem que as propostas de avaliação devam buscar um número sintético de indicadores que permitam ao interessado na avaliação fazer um juízo de valor para subsidiar uma decisão. Com base nessa premissa, e considerando a grande quantidade de dados disponíveis nos sistemas de informação oficiais, a proposta foi desenvolvida com um número reduzido de indicadores, cujas medidas podem ser calculadas mediante dados disponíveis no SIAB e no SIH.

Para analisar a cobertura, calculou-se o percentual de cobertura populacional potencial. Essa opção justifica-se pelo fato de o PSF, originalmente destinado a uma parcela da população, atualmente representar o modelo de atenção primária indicado no país para toda a população. Este indicador é chamado de "cobertura potencial” em função de representar os recursos disponíveis para atender à população para a qual se destina, e não para a qual efetivamente utilizou o PSF.

Para o cálculo da cobertura populacional potencial, utilizou-se a proposta do Ministério da Saúde 25 , na qual o número de residentes no município se dá de acordo com os dados do Instituto Brasileiro de Geografia e Estatística (IBGE), pela fórmula:

$\begin{aligned} & \begin{array}{l}\text { Cobertura } \\ \text { populacional } \\ \text { potencial }\end{array} \\ & \begin{array}{c}\text { Número de residentes } \\ \text { no município }\end{array}\end{aligned} \times 100$

O percentual de cobertura potencial do PSF foi identificado nos dois momentos da pesquisa, bem como sua evolução. A cobertura potencial foi categorizada em: alta (maior que 70\%), média (entre $50 \%$ e $70 \%$ ) e baixa (menor que $50 \%$ ). A evolução foi classificada em aumento, nenhuma alteração, ou diminuição, de acordo com a mudança de categoria entre os anos analisados. Os pontos de corte foram definidos mediante análise de cobertura potencial do PSF no Brasil em 200426.

O indicador "indício de mudança no modelo assistencial" foi medido pelas atividades da equipe de PSF com dados disponíveis nos sis- 
temas de informação. Assim, compuseram esse indicador as visitas domiciliares do médico, os exames de patologia-clínica solicitados, os encaminhamentos ao especialista e o atendimento individual do enfermeiro.

A análise das visitas domiciliares do médico busca compreender o quanto o município valoriza este instrumento de trabalho em saúde da família como agente facilitador do vínculo do profissional médico com a comunidade. Não foram utilizados os dados de visitas domiciliares do profissional de enfermagem, em virtude dessa prática estar mais bem estabelecida entre estes profissionais, e compor sua atuação na atenção primária, mesmo anteriormente ao PSF 27.

Os exames de patologia clínica e os encaminhamentos ao especialista foram incluídos para analisar a inserção do PSF na rede assistencial. Esses procedimentos indicam se o PSF está atuando como porta de entrada do sistema e se permite acesso à média e alta complexidade. Caso contrário, sua atuação é apenas a de um modelo paralelo, destinado a uma parcela da população e com um conjunto pré-determinado e reduzido de procedimentos. Por fim, utilizou-se o indicador de atendimento individual do profissional de enfermagem, pois a construção do trabalho em equipe no PSF pressupõe a participação ativa do enfermeiro na assistência da população adstrita.

As quatro medidas do "indício de mudança de modelo" foram razões cujo numerador é o número médio de consultas médicas individuais realizadas por uma equipe e o denominador é o valor encontrado para o somatório das variáveis descritas acima, no mesmo local e período de tempo. Essa fórmula para as medidas parte do entendimento de que o total de consultas médicas é uma importante representação da assistência à saúde, mas deve estar em equilíbrio com as outras atividades desenvolvidas pela equipe de saúde da família para propiciar mudança no modelo assistencial.

Para o cálculo de consultas médicas esperadas, estabeleceu-se um tempo médio de $20 \mathrm{mi}$ nutos de consulta por paciente, multiplicado por 24 horas de atendimento semanais, totalizando 72 consultas médicas por semana. As 24 horas foram calculadas para um contrato de trabalho de 40 horas, exigido na maior parte dos municípios brasileiros para atuação no PSF; as 16 horas restantes destinam-se às atividades em equipe para promoção, prevenção e reabilitação da saúde, quais sejam: grupos de atenção, visitas domiciliares, educação continuada e planejamento e avaliação das ações.

Para visitas domiciliares do médico, foi considerado o tempo de deslocamento e de maior interação com o paciente em seu domicílio, re- sultando em 40 a 80 minutos por consulta, em 4 horas semanais. Este cálculo estabelece a possibilidade de realização de 3 a 6 visitas domiciliares semanais, que resultaria na proporção de uma visita domiciliar para 12 a 24 consultas médicas.

Até 2005 não estavam estabelecidos parâmetros para exames de patologia clínica e para consultas de enfermagem. Assim, adotaram-se como parâmetros para essas medidas os intervalos entre primeiro e terceiro quartis dos valores encontrados para o Brasil em 2001. A utilização do padrão observado no primeiro período da análise para todo o país foi a alternativa adotada para evitar a utilização de parâmetros teóricos sem comprovação empírica. Desse modo, para os exames de patologia clínica, o valor esperado é de um exame solicitado para 3,1 a 5,4 consultas médicas realizadas. Para atendimentos individuais do profissional de enfermagem, o esperado é de um atendimento para 2,0 a 3,9 consultas médicas realizadas.

Quanto à medida dos encaminhamentos ao especialista, utilizou-se o preconizado pelo Ministério da Saúde para a atenção primária, que é um grau de resolutividade médio de $85 \%$ dos atendimentos 25. Padronizou-se, portanto, um intervalo aceitável de $10 \%$ a $20 \%$ de encaminhamentos, resultando em um encaminhamento para 5,0 a 10,0 consultas médicas realizadas.

O cálculo da variação aceitável dos parâmetros baseou-se na variação das medidas, com o objetivo de respeitar as variações municipais, na forma de implementar e executar as ações preconizadas com base na sua realidade, sem perder de vista a qualidade das atividades da equipe.

Para cada município foi atribuído escore 1 (um) no aspecto analisado cujo valor estivesse dentro do intervalo esperado e escore 0 (zero) para valores acima ou abaixo deste intervalo, uma vez que valores fora do esperado (acima ou abaixo) podem representar desequilíbrio do modelo de atenção, ou uma rede de atenção mal estruturada, com excesso ou falta de oferta de determinados serviços.

Os municípios foram classificados, nos anos de 2001 e 2004, quanto ao modelo assistencial, em três tipos: forte indício de mudança, médio indício de mudança e fraco indício de mudança, de acordo com o somatório dos indicadores alcançados. Quando a soma das medidas foi de 0 a 1 , o município foi classificado, quanto ao modelo assistencial, como em fraco indício de mudança; para soma de valores entre 2 a 3 , a classificação foi de médio indício de mudança; e, no caso de valor 4, a classificação foi de forte indício de mudança.

Os aspectos escolhidos para compor o indício de mudança no modelo assistencial constituem 
apenas uma aproximação possível para medir essa mudança. Outros indicadores poderiam ser utilizados para analisar a mudança do modelo assistencial, tais como os protocolos de atendimento, o acolhimento, a humanização do atendimento. Entretanto, os dados para esses indicadores não podem ser obtidos por meio do SIAB, fato que os exclui da proposta inicial desse estudo de utilizar informações disponíveis nos sistemas.

A análise sistemática de alguns artigos que abordam as internações evitáveis de pacientes por condições sensíveis à atenção ambulatorial, demonstra que pacientes com uma fonte rotineira de cuidados ambulatoriais apresentam um número menor de internações, mesmo controlando a amostra por renda, raça e sexo 28,29,30,31,32.

Assim sendo, optou-se por medir as internações por condições sensíveis à atenção ambulatorial para representar o impacto do PSF. Para tanto, foram utilizados dados secundários disponíveis na página eletrônica do DATASUS sobre morbidade hospitalar, por local de residência, nos anos de 2001 e de 2004. Os dados obtidos foram selecionados com base na lista de morbidade da Classificação Estatística Internacional de Doenças e Problemas Relacionados à Saúde, $10 \underline{a}$ Revisão (CID-10) 33.

As principais causas de internações apontadas por Mendes 13 como sensíveis à atenção primária são: asma (J45), insuficiência cardíaca (I50), pneumonia (J12 a J18), e diarréia e gastroenterite de origem infecciosa presumível (A09). A medida proposta foi a razão entre a soma das internações dessas doenças e o número de pessoas residentes no município, no mesmo período de tempo, multiplicado por mil. Constituiu-se assim uma taxa de internações por doenças sensíveis à atenção primária, para um conjunto de mil habitantes, no período de um ano.

Na literatura ainda não há, de forma consistente, a determinação do valor médio esperado de internações por condições sensíveis à atenção primária. Para classificar os municípios quanto ao impacto, foram utilizados os dados de internações em Santa Catarina, para o ano de 2001. Os valores menores que o primeiro quartil foram considerados como baixo número de internações; os valores entre o primeiro e o terceiro quartil foram considerados como médio número de internações, os maiores que o terceiro quartil foram considerados como alto número de internações, por condições sensíveis à atenção primária. Os valores estão descritos na Tabela 1 .

Esses indicadores compuseram a classificação dos municípios em baixo, médio ou alto grau de implantação do PSF, em 2001 e 2004, comparando-os entre si em cada momento da pesquisa e durante o período estudado, conforme apresentado na Figura 1.

Essas classificações receberam escores diferenciados:

- Escore 5: para alta cobertura, para forte indício de mudança no modelo assistencial e para baixo número de internação por condições sensíveis à atenção ambulatorial.

- Escore 3: para média cobertura, para médio indício de mudança no modelo assistencial e para médio número de internações por condições sensíveis à atenção ambulatorial.

- Escore 0: para baixa cobertura, para fraco indício de mudança no modelo assistencial e para

Tabela 1

Descrição das variáveis, medidas e parâmetros para a classificação dos municípios catarinenses quanto ao Programa Saúde da Família (PSF), 2001 e 2004.

\begin{tabular}{|c|c|c|}
\hline Indicador & Medidas & Parâmetros \\
\hline \multirow[t]{3}{*}{ Cobertura } & Porcentagem de cobertura & $\leq 50 \%$ (baixa cobertura) \\
\hline & populacional potencial & $50 \%$ a $70 \%$ (média cobertura) \\
\hline & & $\geq 70 \%$ (alta cobertura) \\
\hline \multirow[t]{4}{*}{ Indício de mudança } & Visitas domiciliares & 1 visita domiciliar para 12 a 24 consultas médicas * \\
\hline & Exames de patologia clínica & 1 exame de patologia clínica para 3,1 a 5,4 consultas médicas * \\
\hline & Encaminhamento ao especialista & 1 encaminhamento ao especialista para 5,0 a 10,0 consultas médicas * \\
\hline & Atendimento de enfermagem & 1 atendimento de enfermagem para 2,0 a 3,9 consultas médicas * \\
\hline \multirow[t]{3}{*}{ Impacto } & Internações por doenças sensíveis à atenção & $\leq 8,2$ (baixo número de internações) \\
\hline & primária por mil habitantes & $>$ 8,2 e < 18,3 (médio número de internações) \\
\hline & & $\geq 18,3$ (alto número de internações) \\
\hline
\end{tabular}

Fontes: Ministério da Saúde 35 e Departamento de Informática do SUS (DATASUS) 36,37,38,39.

* Os intervalos são os valores esperados para as variáveis. 


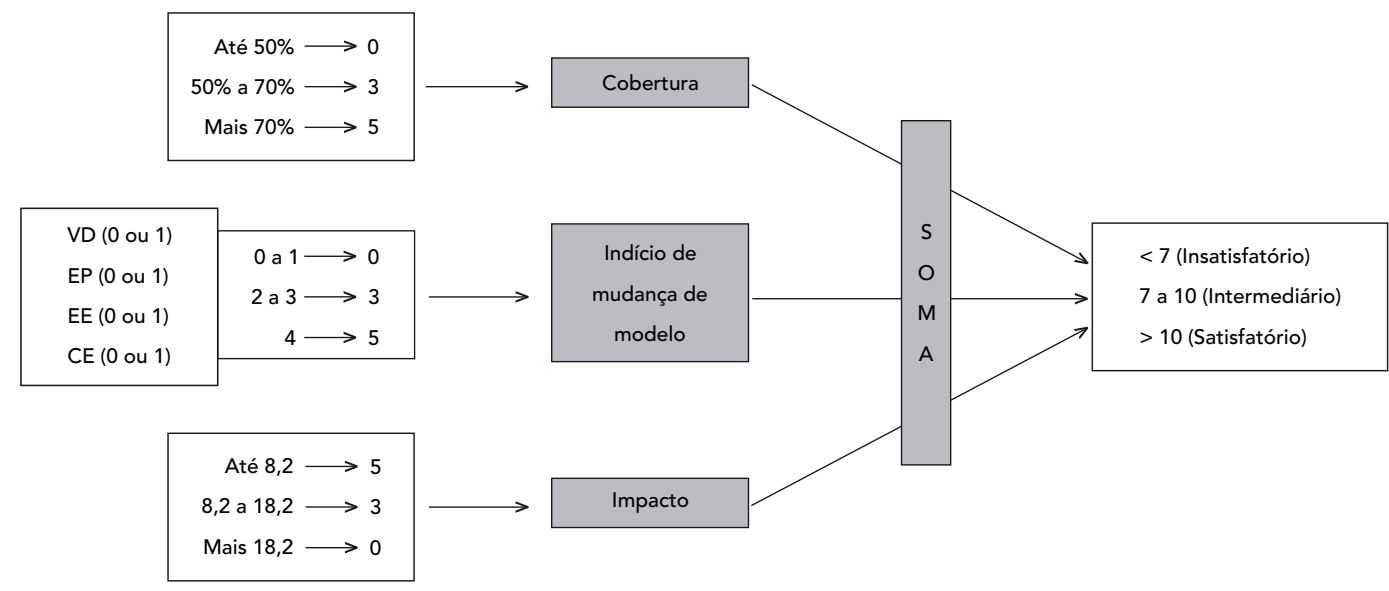

VD: visita médica domiciliar; EP: exames de patologia clínica; EE: encaminhamento ao especialista; CE: consulta de enfermagem.

alto número de internações por condições sensíveis à mudança no modelo assistencial.

A agregação dos escores dos três indicadores analisados permitiu classificar os municípios em três categorias, conforme a implantação do PSF: satisfatória (municípios com somatória de escores maior que 10), intermediária (municípios com somatória de escores de 7 a 10) e insatisfatória (municípios com somatória de escores inferior a 7).

\section{Resultados e discussão}

Os primeiros dados registrados do SIAB de Santa Catarina datam de julho de 1998 e trazem o número de equipes de apenas dois municípios: Chapecó, com nove equipes, e Cocal do Sul, com uma equipe. Em dezembro de 1998 eram 17 os municípios que forneciam dados ao SIAB; tal número aumentou para 46 em dezembro de 1999, e para 175 em dezembro de 2000. Desses últimos, foram eliminados os que não apresentavam os dados necessários para a pesquisa no período de 2000 a 2004. Isto resultou em uma amostra de 125 municípios acompanhados, que representam $42,6 \%$ dos municípios catarinenses.

A descrição e análise dos dados foram realizadas para cada indicador (cobertura, indício de mudança de modelo e impacto) e posteriormen- te para a classificação final, resultado da agregação dos indicadores estudados.

\section{Indicador: cobertura}

Para o ano 2001, foram encontrados os seguintes dados de cobertura populacional potencial: 39 municípios $(31,2 \%)$ com cobertura baixa, 21 $(16,8 \%)$ com cobertura média, e 65 (52\%) com cobertura alta. Para o ano 2004, foram encontrados 17 (13,6\%) com cobertura baixa, 10 (12,5\%) com cobertura média e 98 (78,4\%) com cobertura alta. Os dados podem ser mais bem observados na Tabela 2.

Analisando os dados encontrados nos dois momentos da pesquisa, pode-se observar um aumento significativo ( $\mathrm{p}<0,0001)$ no número de municípios que alcançaram alta cobertura populacional potencial do PSF e uma diminuição naqueles com média e baixa cobertura, para o ano de 2004. Essa ampliação de cobertura do programa nos municípios catarinenses atende a um dos objetivos do programa, que é ampliar o acesso da população aos serviços de saúde.

As mudanças ocorreram em 38 municípios $(30,4 \%)$ no período observado e foram sempre no sentido da ampliação da cobertura: 17 $(13,6 \%)$ mudaram de cobertura baixa para alta; $16(12,8 \%)$ de cobertura média para alta e; 5 (4\%) passaram de cobertura baixa para média. Dos municípios que não apresentaram mudança no 
Municípios catarinenses segundo a classificação dos indicadores: cobertura, indício de mudança de modelo e impacto, para os anos de 2001 e 2004.

\begin{tabular}{|c|c|c|c|c|c|c|}
\hline \multirow[t]{2}{*}{ Indicadores } & \multirow[t]{2}{*}{ Classificação } & \multicolumn{2}{|c|}{2001} & \multicolumn{2}{|c|}{2004} & \multirow[t]{2}{*}{$\mathrm{p}$} \\
\hline & & $\mathbf{n}$ & $\%$ & $\mathbf{n}$ & $\%$ & \\
\hline \multirow[t]{3}{*}{ Cobertura } & Baixa & 39 & 31,2 & 17 & 13,6 & $<0,001$ \\
\hline & Média & 21 & 16,8 & 10 & 8,0 & \\
\hline & Alta & 65 & 52,0 & 98 & 78,4 & \\
\hline \multirow[t]{3}{*}{ Indício de mudança no modelo assistencial } & Fraco & 102 & 81,6 & 102 & 81,6 & 0,599 \\
\hline & Médio & 22 & 17,6 & 23 & 18,4 & \\
\hline & Forte & 1 & 0,8 & - & - & \\
\hline \multirow[t]{3}{*}{ Impacto } & Baixo & 31 & 24,8 & 21 & 16,8 & 0,218 \\
\hline & Médio & 62 & 49,6 & 63 & 50,4 & \\
\hline & Alto & 32 & 25,6 & 41 & 32,8 & \\
\hline
\end{tabular}

Fontes: Ministério da Saúde 35 e Departamento de Informática do SUS (DATASUS) 36,37,38,39.

período, 65 (52\%) tinham cobertura alta, 5 (4\%) tinham média cobertura e 17 (36\%) tinham baixa cobertura

\section{Indicador: indício de mudança no modelo} assistencial

Verificou-se um número maior de municípios que apresentavam número de consultas por visita domiciliar dentro do esperado para o ano de 2001 (29,6\%) em comparação a 2004 (24\%). As consultas por exames de patologia clínica realizados e o percentual de consultas por encaminhamento ao especialista tiveram pouca alteração no período, sem mudanças estatisticamente significativas. A literatura demonstra que a dificuldade de encaminhamento ao especialista interfere na qualidade da atenção à saúde das populações. Nos municípios analisados, constatou-se percentual baixo de encaminhamento ao especialista 6 . Para o indicador de número de consultas por atendimento individual do profissional de enfermagem, observou-se que houve um aumento no percentual de municípios com valores adequados para esta variável. Essa mudança, no entanto, não foi estatisticamente significante $(\mathrm{p}>0,05)$. A Tabela 3 demonstra o percentual de municípios que apresentaram os valores esperados para o ano de 2001 e de 2004.

Ao realizar a soma dos escores dos indicadores, encontramos, para o ano de 2001: um município $(0,8 \%)$ com forte; 22 (17,6\%) com médio e; $102(81,6 \%)$ com fraco indício de mudança. Para o ano de 2004, registraram-se: nenhum município (0\%) com forte; 23 municípios (18,4\%) com médio e; $102(81,6 \%)$ com fraco indício de mu- dança no modelo assistencial. Estes resultados podem ser mais bem observados na Tabela 2 .

A comparação do indicador indício de mudança no modelo assistencial, entre os anos de 2001 e 2004, demonstrou que não existiu diferença significativa no período investigado, indicando que o modelo assistencial não foi alterado com o PSF. Este comportamento foi relatado por Conill 12, que, ao avaliar o PSF no Município de Florianópolis, Estado de Santa Catarina, colocou que as práticas integrais, apesar de terem viabilidade e efeitos positivos, mantêm-se marginalizadas, não sendo suficientes para imprimir mudança no modelo assistencial.

$\mathrm{Na}$ classificação dos municípios quanto à mudança no modelo, encontrou-se que 94 (75,2\%) não apresentaram alteração no período investigado, permanecendo 87 (69,6\%) em fraco e 7 (5,6\%) em médio indício de mudança no modelo assistencial. Houve alteração na classificação de 31 municípios (24,8\%); 15 (12\%) passaram de fraco para médio e o mesmo número passou de médio para fraco, e $1(0,8 \%)$ passou de forte para médio indício de mudança no modelo assistencial.

\section{Indicador: impacto}

Para o ano de 2001, encontrou-se o seguinte resultado: 32 municípios (25,6\%) com baixo; 62 (49,6\%) com médio e; 31 (24,8\%) com alto número de internações por condições sensíveis à atenção primária. Para 2004 os resultados foram: 41 (32,8\%) em baixo número; 63 (50,4\%) em médio número e; 21 (16,8\%) em alto número de internações. Os dados podem ser visualizados na Tabela 2. 
Municípios catarinenses pesquisados com classificação dentro do esperado para consultas por visita domiciliar, exames de patologia clínica, encaminhamento ao especialista, atendimento individual da enfermagem, 2001 e 2004.

\begin{tabular}{|c|c|c|c|c|}
\hline \multirow[t]{2}{*}{ Medida } & \multicolumn{2}{|c|}{2001} & \multicolumn{2}{|c|}{2004} \\
\hline & $\mathbf{n}$ & $\%$ & $\mathbf{n}$ & $\%$ \\
\hline Visita domiciliar & 37 & 29,6 & 30 & 24,0 \\
\hline Exames de patologia clínica & 22 & 17,6 & 24 & 19,2 \\
\hline Encaminhamento ao especialista & 11 & 8,8 & 9 & 7,2 \\
\hline Atendimento individual de enfermagem & 30 & 24,0 & 39 & 31,2 \\
\hline
\end{tabular}

Fontes: Ministério da Saúde 35 e Departamento de Informática do SUS (DATASUS) 36,37,38,39.

Comparando os dados de 2001 e 2004, verificou-se que 75 municípios (60\%) mantiveram sua classificação quanto ao impacto, e 50 (40\%) modificaram sua classificação. Desses, 34 mudaram no sentido da melhoria na classificação: $1(0,8 \%)$ de baixo para alto; 16 (12,8\%) de baixo para médio; e 17 (13,6\%) de médio para alto. Enquanto $16(12,8 \%)$ migraram para uma pior classificação: $9(7,2 \%)$ de alto para médio e $7(5,6 \%)$ de médio para baixo.

Os dados demonstram uma diminuição não significativa na taxa de internações por condições sensíveis à atenção primária no período estudado. Essa variação pode ser aleatória ou relacionada com medidas administrativas de diminuição da liberação de Autorização de Internação Hospitalar (AIH) para os hospitais gerais. Entretanto, pode também representar parcialmente o impacto da reorganização da atenção à saúde causada pelo PSF, que está sendo medida em um curto espaço de tempo. Além disso, o indicador utilizado é facilmente influenciado por pequenas alterações em municípios pequenos.

Tabela 4

Distribuição dos municípios segundo a classificação geral para o Programa Saúde da Família (PSF), 2001 e 2004.

\begin{tabular}{lccccc}
\hline Classificação & & $\mathbf{2 0 0 1}$ & & $\mathbf{2 0 0 4}$ \\
& $\mathbf{n}$ & $\%$ & $\mathbf{n}$ & $\%$ \\
\hline Insatisfatória & 60 & 48,0 & 36 & 28,8 \\
Intermediária & 56 & 44,8 & 73 & 58,4 \\
Satisfatória & 9 & 7,2 & 16 & 12,8 \\
Total & 125 & 100,0 & 125 & 100,0 \\
\hline
\end{tabular}

Fontes: Ministério da Saúde 35 e Departamento de Informática do SUS (DATASUS) 36,37,38,39.

\section{Classificação final dos municípios}

Para classificar os municípios realizou-se a agregação dos escores para os indicadores: cobertura, indício de mudança no modelo assistencial e impacto, classificando-os em três categorias - satisfatória, intermediária e insatisfatória, conforme a Tabela 4 .

Percebeu-se uma mudança significativa ( $\mathrm{p}=$ 0,0061) na classificação final dos municípios para o PSF entre os anos 2001 e 2004, em função de uma redução de 19,2\% no número de municípios que se classificaram como em situação insatisfatória, os quais passaram para a classificação intermediária (13,6\%) e para a situação satisfatória $(5,6 \%)$.

Do total de municípios, $43,2 \%$ mudaram de classificação: $32,8 \%$ para uma melhor classificação e 10,4\% para um pior desempenho em 2004, se comparados com 2001. Somente um município permaneceu em situação satisfatória durante todo o período estudado e $56,8 \%$ do total pesquisado não mudaram de classificação, permanecendo $24,8 \%$ em classificação insatisfatória e $31,2 \%$ em situação intermediária.

\section{Considerações finais}

A análise realizada permitiu conhecer, independentemente e em conjunto, como se comportaram os municípios pesquisados do Estado de Santa Catarina em relação à cobertura, ao indício de mudança e ao impacto da implantação do PSF.

Avaliando a cobertura, percebeu-se que as mudanças operadas entre 2001 e 2004 são estatisticamente significativas e representam uma ampliação em $26,4 \%$ no número de municípios com alta cobertura populacional potencial para o PSF. Esta ampliação significativa na cobertura 
pode ser explicada, entre outros motivos, pelo incentivo financeiro oferecido aos municípios por equipe de PSF implantada. Não é possível afirmar que este programa destina-se somente à parcela mais carente da população, sendo necessários estudos aprofundados acerca do acesso ao programa, para verificar se está sendo utilizado por parcelas distintas da população.

Em relação ao indício de mudança no modelo assistencial, constatou-se um grande número de municípios com fraco indício de mudança no modelo assistencial $(81,6 \%)$ para o ano de 2001, que se mantém no ano de 2004. Isso demonstra que esses municípios catarinenses pesquisados dispunham de um modelo assistencial que não contemplava adequadamente os aspectos analisados, quais sejam, consulta domiciliar do médico, consulta de enfermagem, encaminhamento para especialistas e para exames laboratoriais.

No indicador impacto, verificou-se aumento $(7,2 \%)$ no número de municípios com baixo número de internações preveníveis pela atenção primária. A mudança, contudo, não se mostrou estatisticamente significativa, podendo representar um resultado parcial de mudanças que necessitam de um período maior de tempo para serem observadas.

\section{Resumo}

Esta pesquisa avaliativa teve o propósito de classificar os municípios de Santa Catarina quanto à implantação do Programa Saúde da Família. Foram consideradas três categorias (satisfatória, intermediária e insatisfatória) e dois períodos de análise (2001 e 2004). Os indicadores utilizados foram de cobertura, de indício de mudança no modelo assistência e de impacto. Os resultados demonstraram que a cobertura teve uma alteração significativa entre 2001 e 2004 ( $p<0,0001)$, com ampliação. Os indicadores de indício de mudança no modelo assistencial e de impacto não apresentaram alterações significativas. Para o ano de 2001, observou-se que 48\% dos municípios estavam em situação insatisfatória, 45\% em situação intermediária e 7\% em situação satisfatória, enquanto em 2004 tem-se $29 \%, 58 \%$ e 13\%, respectivamente. Tal alteração, entre 2001 e 2004, no que concerne à melhor classificação, mostrou-se estatisticamente significativa $(p=0,0061)$. Por fim, verificando o conjunto de dados, nota-se que as alterações na classificação final resultaram fundamentalmente do indicador de cobertura, demonstrando a efetiva ampliação do acesso. Entretanto, com esses indicadores não foi possível evidenciar mudanças significativas no modelo assistencial no período analisado.

Programa Saúde da Família; Avaliação em Saúde; Cobertura de Serviços de Saúde
A avaliação da classificação final dos municípios pesquisados identificou mudança estatisticamente significativa no que diz respeito ao aumento do percentual de municípios que obtiveram melhor classificação para o PSF em 2004. No entanto, comparando a classificação final com o resultado dos indicadores analisados, verifica-se que esta mudança se deve fundamentalmente ao aumento da cobertura populacional potencial, uma vez que o indicador "indício de mudança no modelo assistencial” não mostrou alteração, e o indicador impacto mostrou uma alteração não significativa.

Estes dados fazem supor que o PSF, nos municípios estudados de Santa Catarina, apresentou significativa ampliação de sua cobertura populacional potencial, mas carecia de qualificação para mudança do modelo assistencial e para obter resultados expressivos. Essa constatação é coerente com o pequeno número de profissionais adequadamente preparados para o trabalho em equipe e para abordagem integral em saúde. Estudos futuros poderão avaliar o impacto das propostas mais recentes para superar o problema, como as especializações e residências em saúde da família e as ações desenvolvidas pelo Projeto de Expansão e Consolidação do Saúde da Família (PROESF) 34 .

\section{Colaboradores}

F. Henrique foi responsável por coletar os dados e fazer a revisão da literatura. F. Henrique e M. C. M. Calvo delinearam o estudo, analisaram os dados, escreveram e fizeram a revisão final do artigo. 


\section{Referências}

1. Arouca AS. O dilema preventivista: contribuição para a compreensão e crítica da medicina preventiva [Tese de Doutorado]. Campinas: Universidade Estadual de Campinas; 1975.

2. Donnangelo MCF, Pereira L. Saúde e sociedade. São Paulo: Editora Duas Cidades; 1976.

3. Starfield B. Atenção primária: equilíbrio entre a necessidade de saúde, serviços e tecnologias. Brasília: Organização das Nações Unidas para a Educação, a Ciência e a Cultura/Ministério da Saúde; 2002.

4. Testa M. Pensar em saúde. Porto Alegre: Editora Artes Médicas/Rio de Janeiro: ABRASCO; 1992.

5. Ministério da Saúde. Programa Saúde da Família: saúde dentro de casa. Brasília: Ministério da Saúde; 1994.

6. Viana ALDA, Dal Poz MR. A reforma sanitária do sistema de saúde no Brasil e Programa Saúde da Família. Physis (Rio J) 1998; 2:225-64.

7. Ministério da Saúde. Saúde da família: uma estratégia para reorientação do modelo assistencial. Brasília: Ministério da Saúde; 1997.

8. Ministério da Saúde. Avaliação da implementação do Programa Saúde da Família em dez grandes centros urbanos. Brasília: Ministério da Saúde; 2004.

9. Ministério da Saúde. Avaliação normativa do Programa Saúde da Família no Brasil. Brasília: Ministério da Saúde; 2002

10. Misoczky MC. A medicina de família, os ouvidos do príncipe e os compromissos com o SUS. Saúde Debate 1994; 42:40-4.

11. Aguiar, DS. A "saúde da família" no Sistema Único de Saúde: um novo paradigma? [Dissertação de Mestrado]. Rio de Janeiro: Escola Nacional de Saúde Pública, Fundação Oswaldo Cruz; 1994.

12. Conill EM. Políticas de atenção primária e reformas sanitárias: discutindo a avaliação a partir da análise do Programa Saúde da Família em Florianópolis, Santa Catarina, Brasil, 1994-2000. Cad Saúde Pública 2002; 18 Suppl:S191-202.

13. Mendes EV. A atenção primária à saúde no SUS. Fortaleza: Escola de Saúde Pública do Ceará; 2002.

14. Ronzani TM, Stralen CJ. Dificuldades de implantação do Programa Saúde da Família como estratégia de reforma do sistema de saúde brasileiro. Revista de Atenção Primária à Saúde 2003; 2:99-107.

15. Viana ALd'A, Rocha JSY, Elias PE, Ibañez N, Moraes MHD. Modelos de atenção básica nos grandes municípios paulistas: efetividade, eficácia, sustentabilidade e governabilidade. Ciênc Saúde Coletiva 2006; 11:577-606

16. Ibañez N, Rocha JSY, Castro PC, Ribeiro MCSA, Forster AC, Novaes MHD, et al. Avaliação do desempenho da atenção básica no Estado de São Paulo. Ciênc Saúde Coletiva 2006; 11:683-703.

17. Elias PE, Ferreira CN, Alves MCG, Cohn A, Kishima V, Escrivão Junior A, et al. Atenção básica em saúde: comparação entre PSF e UBS por estrato de exclusão social no município de São Paulo. Ciênc Saúde Coletiva 2006; 11:633-41.
18. Facchini LA, Piccini RX, Tomasi E, Thumé E, Silveira DS, Siqueira FV, et al. Desempenho do PSF no Sul e no Nordeste do Brasil: avaliação institucional e epidemiológica da atenção básica à saúde. Ciênc Saúde Coletiva 2006; 11:669-81.

19. Santos R. Saúde da família: possibilidade e limites para viabilização do PSF na região do Monte Cristo [Monografia de Graduação]. Florianópolis: Universidade Federal de Santa Catarina; 1998.

20. Secretaria de Estado da Saúde de Santa Catarina. Estratégia do Programa Saúde da Família - PSF e Programa Agentes Comunitários - PCS no Estado de Santa Catarina. http://www.saude.sc.gov.br/ PSF/Textos/Estrat\%E9gia\%20Programa\%20Sa\%FA de\%20da\%20Fam\%EDlia\%20e\%20Programa\%20d e\%20Agentes\%20Comunit\%E1rios\%20-\%20PACS. doc (acessado em 07/Mar/2005).

21. França T. Sistema de Informação da Atenção Básica: um estudo exploratório [Dissertação de Mestrado]. Rio de Janeiro: Escola Nacional de Saúde Pública, Fundação Oswaldo Cruz; 2001.

22. Ministério da Saúde. Manual do SIAB: Sistema de Informação da Atenção Básica. Brasília: Ministério da Saúde; 2003.

23. Donabedian A. The quality of care: how can it be assessed? JAMA 1988; 260:1743-8.

24. Contandriopoulos AP, Touati N, Denis JL, Rodriguez R, Sicotte C, Nguyen H. Uma experiência de integração dos médicos em uma zona rural: os desafios da implantação. In: Hartz ZMA, Silva LMV, organizadoras. Avaliação em saúde: dos modelos teóricos à prática na avaliação de programas e sistemas de saúde. Salvador: Editora da Universidade Federal da Bahia/Rio de Janeiro: Editora Fiocruz; 2005. p. 255-75.

25. Ministério da Saúde. Guia prático do Programa Saúde da Família. Brasília: Ministério da Saúde; 2001.

26. Departamento de Informática do SUS. Indicadores de cobertura. http://tabnet.datasus. gov.br/ cgi/tabcgi.exe?idb2002/f02.def (acessado em 19/ Mar/2005).

27. Albiero CE. Assistência domiciliar como modalidade de atendimento à saúde no contexto da família e das políticas sociais [Dissertação de Mestrado]. Florianópolis: Programa de Pós Graduação em Saúde Pública, Universidade Federal de Santa Catarina; 2003.

28. Weissman JS, Gatsonis C, Epstein AM. Rates of avoidable hospitalization by insurance status in Massachusetts and Maryland. JAMA 1992; 268:2388-94.

29. Fylkesnes K. Determinants of health care utilization - visits and referrals. Scand J Soc Med 1993; 21:325-32.

30. Bindman AB, Grumbach K, Osmond D. Preventable hospitalizations and access to health care. JAMA 1995; 274:305-11.

31. Kupor SA, Liu YC, Lee J. The effect of copayments and income on the utilization of medical care by subscribers to Japan's National Health Insurance System. Int J Health Serv 1995; 25:295-312. 
32. Halfon N, Newacheck PW, Wood DL, St Peter RF. Routine emergency department use for sick care by children in the United States. Pediatrics 1996; 98:28-34.

33. Organização Mundial da Saúde. Classificação estatística internacional de doenças e problemas relacionados à saúde, $10^{\mathrm{a}}$ revisão. São Paulo: Centro Colaborador da OMS para a Classificação de Doenças em Português; 1995.

34. Departamento de Atenção Básica, Ministério da Saúde. Apoio à conversão do modelo de atenção básica à saúde. http://dtr2002.saude.gov.br/ proesf/Site/Paginas/ComponenteI.htm (acessado em 28/Ago/2007).

35. Ministério da Saúde. Agentes comunitários de saúde, equipes de saúde da família, equipes de saúde bucal em atuação. Competência: setembro de 2004. http://www.saude.gov.br (acessado em 02/Nov/2004).
36. Departamento de Informática do SUS. Produção e marcadores. http://www.datasus.gov.br/siab/ siabp.htm?saude=http\%3A\%2F\%2Fwww.datasus. gov.br\%2Fsiab\%2Fsiabp.htm\&obj=\%24VObj\&bota ook=OK (acessado em 18/Mai/2005).

37. Departamento de Informática do SUS. Cadastramento familiar. http://www.datasus.gov.br/siab/ siabp.htm?saude=http\%3A\%2F\%2Fwww.datasus gov.br\%2Fsiab\%2Fsiabp.htm\&obj=\%24VObj\&bota ook=OK (acessado em 22/Jun/2005).

38. Departamento de Informática do SUS. População residente. http://tabnet.datasus.gov.br/cgi/ deftohtm.exe?ibge/cnv/popsc.htm (acessado em 22/Jun/2005).

39. Departamento de Informática do SUS. Morbidade hospitalar. http://tabnet.datasus.gov.br/cgi/ deftohtm.exe?sih/cnv/mrsc.htm (acessado em 09/ $\mathrm{Jul} / 2005)$.

Recebido em 17/Jul/2006

Versão final reapresentada em 11/Set/2007

Aprovado em 20/Set/2007 